$12-1-1998$

\title{
Relaxational Mode Structure for Optical Probe Diffusion in High Molecular Weight Hydroxypropylcellulose
}

Kiril A. Streletzky

Cleveland State University, K.STRELETZKY@csuohio.edu

George D.J. Phillies

Worcester Polytechnic Institute, phillies@wpi.edu

Follow this and additional works at: https://engagedscholarship.csuohio.edu/sciphysics_facpub

Part of the Physics Commons

How does access to this work benefit you? Let us know!

\section{Publisher's Statement}

This is the accepted version of the following article: Kiril A. Streletzky and George D. J. Phillies, "Relaxational mode structure for optical probe diffusion in high molecular weight hydroxypropylcellulose," Journal of Polymer Science Part B: Polymer Physics 36 (17), 3087-3100 (1998). , which has been published in final form at http://onlinelibrary.wiley.com/doi/ 10.1002/(SICI)1099-0488(199812)36:17\%3C3087::AID-POLB9\%3E3.0.C0;2-2/pdf

\section{Repository Citation}

Streletzky, Kiril A. and Phillies, George D.J., "Relaxational Mode Structure for Optical Probe Diffusion in High Molecular Weight Hydroxypropylcellulose" (1998). Physics Faculty Publications. 261.

https://engagedscholarship.csuohio.edu/sciphysics_facpub/261

This Article is brought to you for free and open access by the Physics Department at EngagedScholarship@CSU. It has been accepted for inclusion in Physics Faculty Publications by an authorized administrator of EngagedScholarship@CSU. For more information, please contact library.es@csuohio.edu. 


\section{Relaxational Mode Structure for Optical Probe Diffusion in High Molecular Weight Hydroxypropylcellulose}

KIRIL A. STRELETZKY, GEORGE D. J. PHILLIES

\section{INTRODUCTION}

The mechanism of polymer dynamics in solutions is a major unanswered question that has been studied intensively for many years. There are many different experimental approaches to the problem. ${ }^{1}$ One of the widely used techniques is the optical probe diffusion experiment. In this kind of experiment one observes the motion of dilute monodisperse probe particles through the polymer solution of interest. If probes scatter considerably $(\sim 99 \%)$ more light than the polymer does, then one can obtain crucial information about the polymer dynamics by monitoring the diffusion of probe particles. Optical probe diffusion can be observed by a number of experimental techniques. Our laboratory studies probe diffusion using quasi-elastic light-scattering spectroscopy (QELSS), which infers the dynamic structure factor $S(q, t)$ of the scattered light.

The use of the optical probe technique was pioneered by Turner and Hallett ${ }^{2}$ more than 20 years ago. Since then numerous experimental studies in different polymer solutions ${ }^{3-17}$ have revealed a wide variety of probe diffusion phenomenologies.

It was demonstrated ${ }^{18,19}$ that in systems in which the functional form of $S(q, t)$ is close to a simple exponential one can obtain the probe's self-diffusion coefficient $D_{p}$ from $S(q, t)$. One can 
then compare $D_{p}$ with the macroscopic zero-shear viscosity $\eta$ of the solution by using the StokesEinstein equation

$$
D=\frac{k_{B} T}{6 \pi \eta R_{h}}
$$

Here $k_{B}$ is Boltzmann's constant, $T$ is the absolute temperature, and $R_{h}$ is the hydrodynamic radius of the diffusing probe. Equation (1) is known to work for mesoscopic probe diffusion in low-viscosity small-molecule solvents. Equation (1) does not always work in high-viscosity largemolecule solvents. ${ }^{3-6}$ However, in some systems $S(q, t)$ is highly bimodal, posing the question of how a single diffusion coefficient $D_{p}$ can represent two decay modes in $S(q, t)$. An alternative detailed lineshape analysis of $S(q, t)$ is needed in this case.

This article addresses the results of a study ${ }^{20}$ of probe diffusion in aqueous solutions of hydroxypropylcellulose. The scattering from probes diffusing in this polymer solution reveals a bimodal $S(q, t)$. Lineshape analysis of $S(q, t)$ was employed $^{20}$ for this system, finding two distinct modes of the relaxation. An apparent influence of the probe size on the relaxational mode structure was discovered. ${ }^{20}$ The detailed lineshape analysis can be found in ref. 20. Here we discuss properties of the spectral parameters that reveal the physical nature of the relaxational mode structure. In our analysis of the physical nature of modes, we also attempted to apply the coupling/scaling mod$\mathrm{el}^{21}$ analysis to our system. ${ }^{22}$

The following sections introduce experimental methods, give the important results of ref. 20, present a coupling/scaling model analysis of our data, and discuss the modes' physical interpretations.

\section{EXPERIMENTAL}

Quasi-elastic light-scattering spectroscopy studies the temporal evolution of concentration fluctuations in a sample by monitoring the light-scattering intensity $I(q, t)$ (where $q$ is the scattering vector) and calculating the intensity-intensity correlation function:

$$
S(q, \tau)=\int_{0}^{T} d t I(q, t) I(q, t+\tau) .
$$

Here, $\tau$ is a shift in time, and $T$ is the duration of the experiment. Our light-scattering spectrometer used a $1.5 \mathrm{~W} \mathrm{Ar}^{+}$(Spectra-Physics 2020-03) CW laser coupled to a photometergoniometer (Brookhaven Instruments BI-200SM). Sample cells, placed into a decalin-filled index-matching vat, were maintained at $25 \pm 0.1^{\circ} \mathrm{C}$. Spectra were analyzed with a 270-channel digital multitau correlator (Brookhaven Instruments BI2030AT).

The subject of this study was hydroxypropylcellulose (HPC), nominal molecular weight $1 \mathrm{MDa}$ (Scientific Polymer Products), in aqueous solution. Stock solutions of polymer concentration 7 $\mathrm{g} / \mathrm{L}$ were prepared in purified water (Millipore Milli-RO, Milli-Q water systems) and diluted to cover polymer concentrations of $0-7 \mathrm{~g} / \mathrm{L}$ generally at $1 \mathrm{~g} / \mathrm{L}$ intervals. HPC absorption by probes was prevented by adding a trace amount (based on Phillies et al. ${ }^{23}$ ) of surfactant [0.2 wt \% TX-100 (Aldrich)]. Carboxylate-modified polystyrene latex spheres (PSL) with nominal diameters of 14 , $21,38,67,87,189,282$, and $455 \mathrm{~nm}$ (Interfacial Dynamics, Seradyn, Dow Chemicals) were used in the probe diffusion experiments. Because latex spheres are very good scatterers, even trace amounts of PSL $(5-10 \mu \mathrm{L}$ per $\mathrm{mL}$ of polymer solution) were enough for probes to dominate the scattering intensity. Probe multiple scattering was avoided by using small probe volume fractions (under 0.001).

The observed spectra in our experiments correspond to probe motion through the practically unseen polymer matrix. There is no sizable contribution from concentration fluctuations of polymer or surfactant. To test this point we directly compared spectra of probe-containing and probefree polymer solutions under identical operating conditions. Typically $S(q, 0)$ of a probe-free polymer solution is about $1 \%$ of $S(q, 0)$ of a probecontaining polymer solution: the probe contribution to the total scattering is far greater than any other contribution. We also subtracted (at the field-correlation function level) spectra of probefree polymer solutions from spectra of probe-containing polymer solutions to find the contribution of the polymer scattering. The difference spectra then were subjected to our spectral fitting routine, yielding the same fitting parameters (within experimental error) as did unsubtracted probecontaining spectra.

Analyzing our spectra, we found that they were highly bimodal. A calculation of the probe diffusion coefficient $D_{p}$ from the initial logarithmic slope of $S(q, t)$ therefore does not describe the 
long-time part of the spectrum. A systematic analysis of the spectral lineshape is required.

We analyzed the spectral lineshape on the level of field correlation function $g^{(1)}(q, t)$. The intensity-intensity correlation function $g^{(2)}(q, t)$ is related to $g^{(1)}(q, t)$ via

$$
g^{(2)}(q, t)=S(q, t)-B=A\left(g^{(1)}(q, t)\right)^{2},
$$

where $A$ is the scattering amplitude and $B$ is the baseline, the time-independent part of the spectrum. We tried different forms of $g^{(1)}(q, t)$ to see which one best described $g^{(2)}(q, t)$. We minimized $\left[g^{(2)}(q, t)-S(q, t)\right]^{2} /[S(q, t)]^{2}$, applying nonlinear least squares and the simplex algorithm. ${ }^{24}$

\section{RESULTS}

This section presents a generalized analysis of our light-scattering spectra and physical properties of the modes that were found to compose our spectra. A more detailed description of our findings is found in ref. 20.

Analyzing highly nonexponential $S(q, t)$ of probes of different diameter $d$ we found ${ }^{20}$ that probes with $d<67 \mathrm{~nm}$ clearly have bimodal spectra. Even though spectra of probes with $d$ $\geq 67 \mathrm{~nm}$ do not demonstrate an obvious bimodal lineshape to the eye, they are also found by numerical analysis to be bimodal. The bimodal $g^{(1)}(t)$ that describes our spectra has the form

$g^{(1)}(t)=\left(1-A_{f}\right) \exp \left(-\theta t^{\beta}\right)+A_{f} \exp \left(-\theta_{f} t^{\beta_{f}}\right)$.

Here, $\theta, \beta$ are the relaxation pseudorate and stretching exponent of the slow mode, $\theta_{f}, \beta_{f}$ are the relaxation pseudorate and stretching exponent of the fast mode, and $A_{f}$ is the amplitude fraction of the fast mode. The visible difference in the lineshape of $S(q, t)$ between small $(d<67$ $\mathrm{nm})$ and large $(d \geq 67 \mathrm{~nm})$ probes is reflected in different values of the slow stretching exponent $\beta$. For large probes, forcing $\beta \equiv 1$ produced ${ }^{20}$ much more stable and reproducible fits then did allowing $\beta$ to be a free parameter. For small probes, fits with $\beta \approx 0.7-0.95$ gave excellent results.

Our analysis decomposes spectra of polystyrene sphere probes into two modes, a fast stretched-exponential mode and a slower exponential or stretched-exponential mode. It should be emphasized that our interpretation of the modes as stretched exponentials is phenomeno- logical. A group of exponential modes whose sum approximates a stretched exponential decay cannot, within the limits of our experimental method, be distinguished from a single stretched-exponential mode. Our remarks on the properties of single modes may, therefore, actually be statements about the aggregate behavior of a group of modes. However, sums of two pure exponentials do not fit our spectra. A single stretched-exponential mode may be a stretched exponential or an aggregate of pure exponentials, but is observably not a single pure exponential.

Summarizing, in our lineshape analysis we found ${ }^{20}$ slightly different but bimodal (fast and slow modes) spectral lineshapes for small and for large probes. Probes with $d<67 \mathrm{~nm}$ have $g^{(1)}$ in the form of two stretched exponentials, while probes with $d \geq 67 \mathrm{~nm}$ have $g^{(1)}$ in the form of a fast stretched and a slow pure exponential. The difference ${ }^{20}$ in the functional description of $g^{(1)}(t)$ between large and small probes remained the same at all polymer concentrations studied. The crossover probe radius separating small and large probe behavior $^{20}$ is comparable with the chain dimensions. In particular, small probes are smaller than the hydrodynamic radius of the polymer, while large probes are comparable or larger than the radius of gyration of the polymer. Our spectra thus may be divided naturally by a time scale and probe size into four regimes, namely two modes (fast and slow) for small $\left(d<R_{h}\right)$ probes, and two modes for large $(d$ $\geq R_{g}$ ) probes.

The difference between large and small probes is made very prominent if one considers the diameter dependence of the pseudorates $\theta$ and $\theta_{f}$. Figure 1(a) gives the probe diameter dependence of $\theta$ for each polymer concentration studied. There are two regimes: (1) small probes with no concentration dependence of $\theta$, and (2) large probes with $\theta$ strongly depending on $c$. The transition from small- to large-probe behavior begins as $d$ approaches the hydrodynamic radius $R_{h}$ of the chain, and is complete for $d$ larger than the radius of gyration $R_{g}$ of the chain. This length scale remains the same for polymer concentrations up to $c=7 \mathrm{~g} / \mathrm{L}$, which corresponds to $c[\eta]=5$. The concentration independence of the transition length scale is inconsistent with some transient gel models. The typical transient gel models ${ }^{25,26}$ consider the distance between the chain entanglements $\xi$ as the length scale that influences the probe motion through the polymer. The polymer solution, according to these models, would act like 


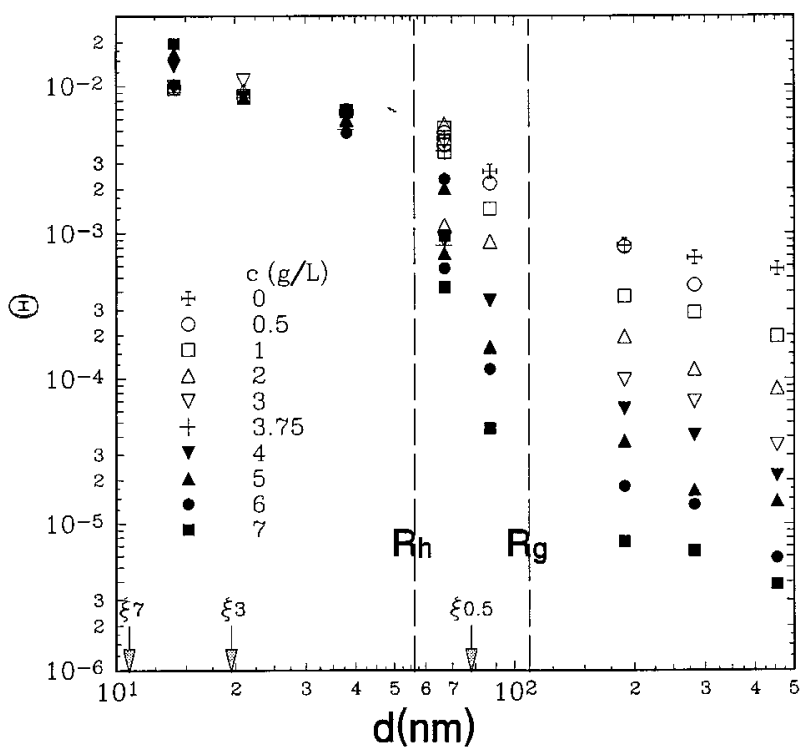

(a)

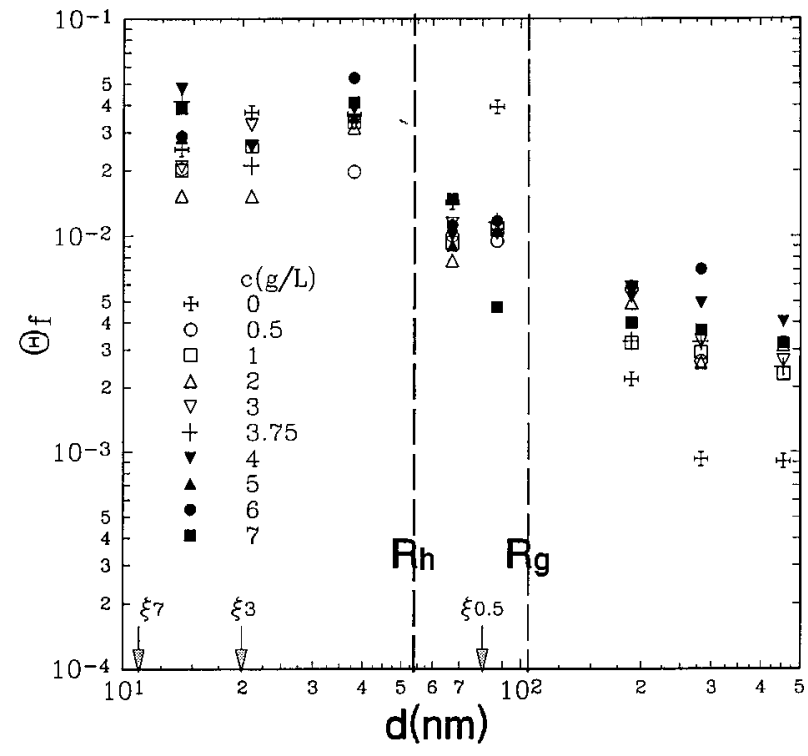

(b)

Figure 1. Probe diameter dependences of the relaxation pseudorates $\theta$ and $\theta_{f}$ [eq. (4)] at various indicated concentrations $c$ for different modes of small and large probes in solutions of $1 \mathrm{MDa}$ HPC: (a) $\theta$ for the slow mode; and (b) $\theta_{f}$ for the fast mode. Vertical dashed lines indicate the hydrodynamic radius $R_{h}$ and the radius of gyration $R_{g}$ of the polymer. Arrows indicate the mesh size $\xi$ calculated as $R_{g}\left(\frac{c}{c^{*}}\right)^{-3 / 4}$, where $c^{*}$ is an overlap concentration.

a net with a mesh size $\xi$, allowing small probes with radius $R<\xi$ to diffuse through the polymer net following the solvent flow, and effectively trapping large probes with $R>\xi$, forcing them to move with the macroscopic viscosity of the solution. However, these models define ${ }^{26} \xi$ as $R_{g}$ at the overlap concentration $c^{*}$ and predict a strong concentration dependence of $\xi$ in the form $\xi=R_{g}$ $\left(\frac{c}{c^{*}}\right)^{-3 / 4}$. Therefore, according to the transient gel models, the separation length scale between small- and large-probe regimes should be a $c$ dependent mesh size $\xi$, which directly contradicts our finding that the transition length scale is independent of $c$. On the other hand, the transition region location at $\left(R_{h}, R_{g}\right)$ and its independence from the polymer concentration is consistent with the fundamental importance of hydrodynamic interactions in probe diffusion.

A similar transition in probe behavior can be seen in Figure 1(b), which plots $\theta_{f}$ vs. $d$. Again, there are two probe regimes: (1) small $\left(d<R_{h}\right)$ probes with a $\theta_{f}$ that is independent of $d$, and (2) large $\left(d>R_{g}\right)$ probes with a $\theta_{f}$ that weakly decreases (within a factor of 2) with increasing $d$. In the transition regime $R_{h}<d<R_{g}$, as $d$ is increased from 40 to $180 \mathrm{~nm}, \theta_{f}$ falls by a factor of 10. However, in both the large- and the smallprobe regimes, $\theta_{f}$ is largely $c$ independent.

Figure 2 gives the concentration dependence of $\theta$ and $\theta_{f}$ for probes of all sizes. As seen in Figure 2(a), for small probes, $\theta$ is largely concentration independent, while for large probes $\theta$ decreases with increasing $c$, closely following

$$
\theta=\theta_{o} \exp \left(-\alpha c^{\nu}\right)
$$

Here, $\alpha$ is a scaling prefactor, $\nu$ is a scaling exponent, and $\theta_{0}$ is the intercept. Figure 2(b) shows the concentration dependence of $\theta_{f}$. $\theta_{f}$ of both small and large probes is largely $c$ independent (within factor of 2), just as $\theta$ of the small-probe slow mode was independent of $c$.

From the $c$ dependence of $\theta$, one can separate the slow modes of large and small probes from each other. For the fast mode, the $c$ dependences of $\theta_{f}$ of small and large probes are very similar. However, the $d$ dependence of $\theta_{f}$ [Fig. 1(b)] allows one to separate the small- and large-probe fast modes. The similarities in the concentration dependences of three out of four modes raises the 


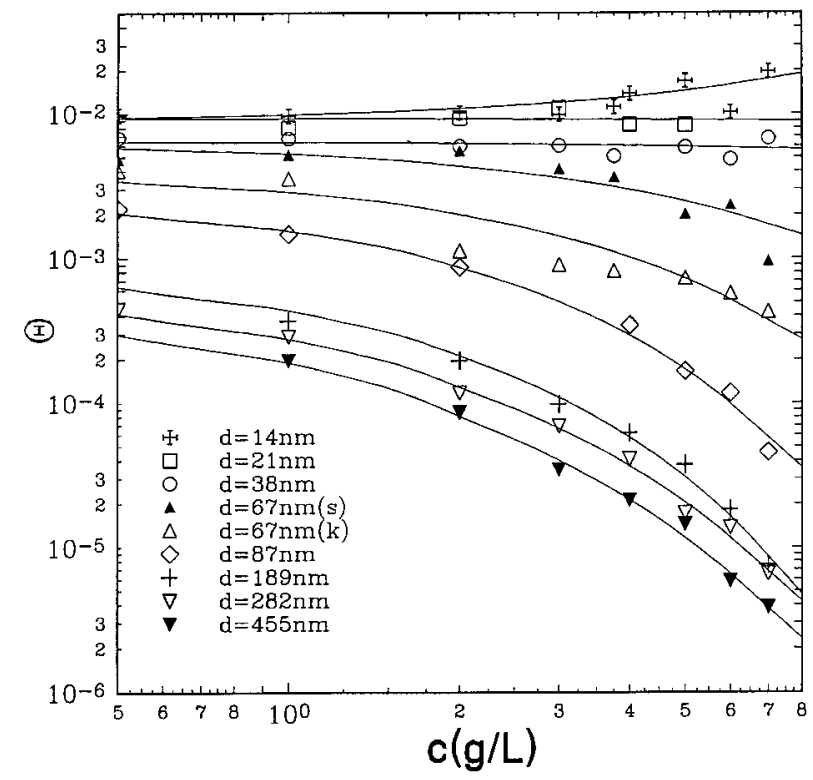

(a)

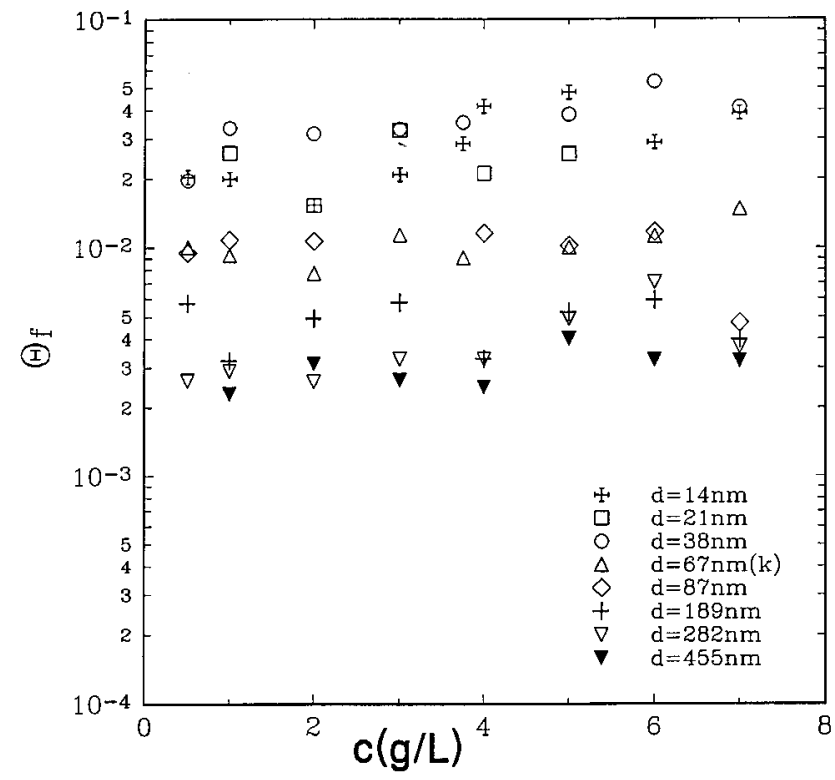

(b)

Figure 2. Concentration dependences of the relaxation pseudorates $\theta_{f}$ and $\theta_{f}$ [eq. (4)] for small $(d \leq 67 \mathrm{~nm})$ and large $(d \geq 67 \mathrm{~nm})$ probes in solutions of 1 MDa HPC: (a) $\theta$ of the slow mode; and (b) $\theta_{f}$ of the fast mode. Solid lines on Figure 2(a) are stretched exponentials in $c$. Units of $\theta$ are $(\mu S)^{-\beta}$.

question of the relation of the modes to each other. If one compares the absolute values of $\theta$ and $\theta_{f}$ for all three modes, one finds that the four modes have only three time scales, namely: (1) the large-probe slow mode with $\theta \sim 10^{-3}-5$ $\times 10^{-6}$, (2) the large-probe fast mode and the small-probe slow mode with $\theta_{f}$ or $\theta \sim 1 \times 10^{-2}-3$ $\times 10^{-3}$, and (3) the small-probe fast mode with $\theta_{f}$ $\sim 5 \times 10^{-2}$.

Mode time scales may also be characterized by relaxation times $\tau=\theta^{-1 / \beta}$ and $\tau_{f}=\theta_{f}^{-1 / \beta_{f}}$. $\tau$ and $\tau_{f}$ have dimensions of (time), ${ }^{1}$ so they are inequivalent to $\theta$ and $\theta_{f}$, which have dimensions $(\text { time })^{-\beta}$. For four mode/probe-size combinations, $\tau$ and $\tau_{f}$ are: (a) $\tau \sim 10^{-3}-3 \times 10^{-1} \mathrm{~s}$ for the large-probe slow mode, (b) $\tau_{f} \sim 6 \times 10^{-4}$ $10^{-1} \mathrm{~s}$ for the large-probe fast mode, (c) $\tau$ $\sim 10^{-4}-8 \times 10^{-4} \mathrm{~s}$ for the small-probe slow mode, and (d) $\tau_{f} \sim 3 \times 10^{-4}-5 \times 10^{-2} \mathrm{~s}$ for the small-probe fast mode.

The longest time scale involves the large-probe slow mode, which has the largest $\tau$. We can rationally incorporate the large-probe fast mode $\left(\tau_{f}^{\text {large }}\right)$ and the small-probe slow mode $\left(\tau^{\text {small }}\right)$ into a single intermediate time scale. The small-probe fast mode, while strongly scattered, usually satisfies $\tau_{f}^{\text {large }} \geq \tau_{f}^{\text {small }} \geq \tau^{\text {small, }}$ for small probes $\tau_{f}$ $>\tau$ even though $\theta_{f}>\theta$. This difference between $\tau$ and $\theta$ arises because for small probes $\beta \approx 0.5-$ 0.9 but $\beta_{f} \approx 0.2-0.5$. It is legitimate to inquire whether we should interchange $\theta$ and $\theta_{f}$ for the small spheres; indeed, on a plot of $g^{(1)}(q, t)$ the visible decay of $\exp \left(-\theta t^{\beta}\right)$ occurs before the visible decay of $\exp \left(-\theta_{f} t^{\beta_{f}}\right)$, because the relative values of $\beta$ and $\beta_{f}$ cause the slowest of the fastmode components to decay at later times than the slowest slow mode component decay. However, as seen in Figures 1 and 2, $\theta$ and $\theta_{f}$ have smooth dependences on $d$ and $c$. If one were to interchange $\theta$ and $\theta_{f}$ of the small spheres, Figures 1 and 2 would show that $\theta$ and $\theta_{f}$ each had nonmonotonic dependences on $d$ and $c$. It is, therefore, rational to label the small-probe $\left(\theta_{f}, \beta_{f}\right)$ mode as the "fast" process, even though $\tau_{f}^{\text {small }}$ $\geq \tau^{\text {small }}$.

We now consider the physical properties of the modes, in terms of a three time scale description of the system:

\section{Mode Concentration Dependences}

First, we compare the concentration dependences of $\theta$ and $\theta_{f}$ with the concentration dependence of the zero-shear viscosity $\eta$. Figure 3 shows the $c$ dependences of $\theta \eta$ [Fig. 3(a)] and $\theta_{f} \eta$ [Fig. 3(b)]. $\eta$ 


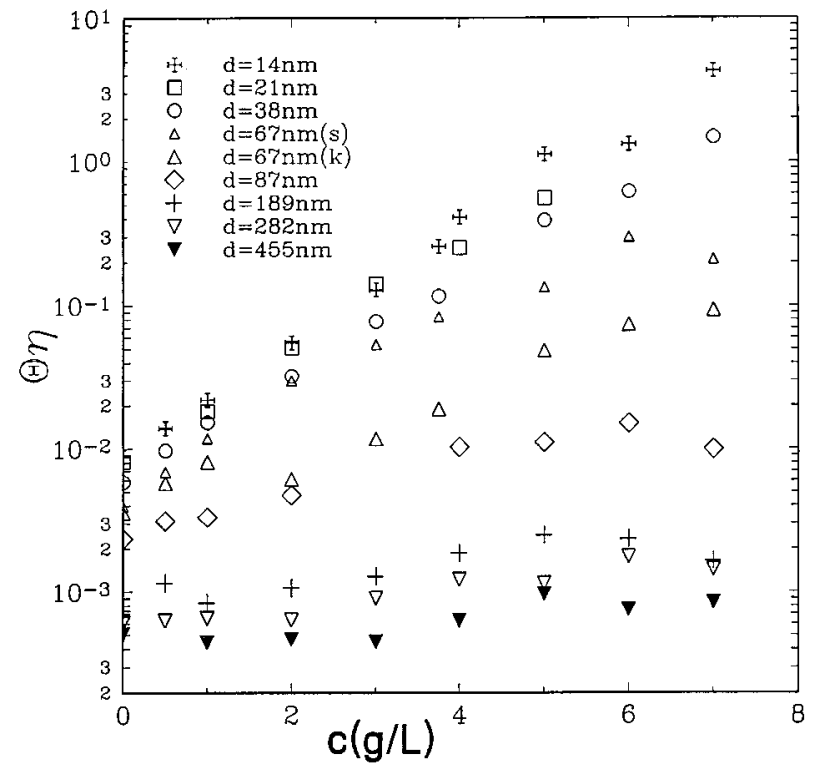

(a)

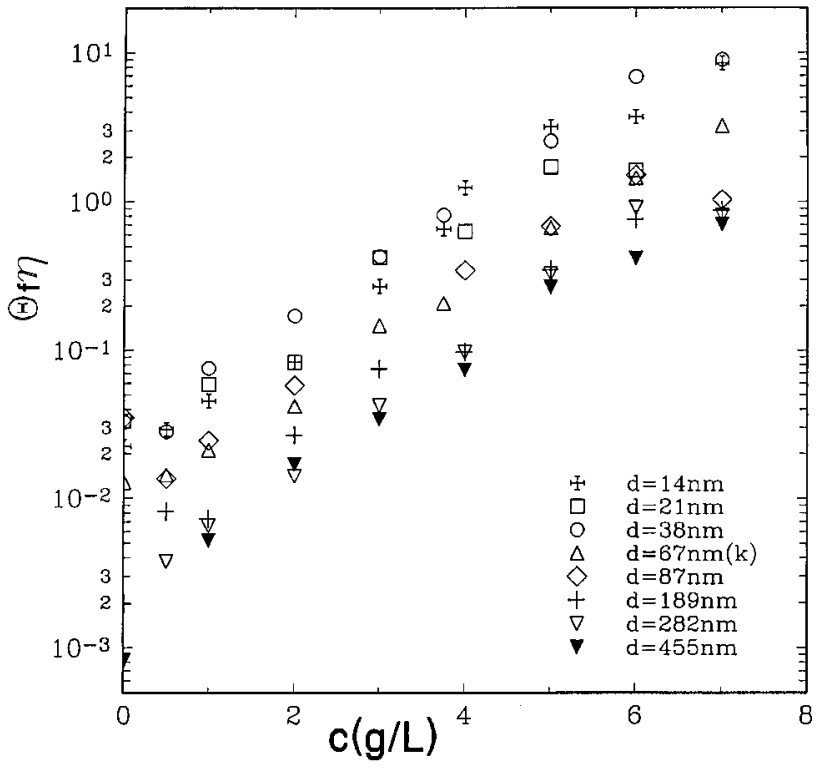

(b)

Figure 3. Concentration dependences of the product of the relaxation pseudorate and solution viscosity for small ( $d \leq 67 \mathrm{~nm})$, and large $(d \geq 67 \mathrm{~nm})$ probes in solutions of $1 \mathrm{MDa}$ HPC: (a) $\theta \eta$ of the slow mode; (b) $\theta_{f} \eta$ of the fast mode. $\theta$ and $\theta_{f}$ are from eq. (4). $\eta$ is from ref. 27.

is from the viscosity measurements of Quinlan and Phillies. ${ }^{27}$ Consider the slow mode [Fig. 3(a)]: (1) for small probes $(d \leq 67 \mathrm{~nm})$ : $\theta$ does not track $\eta^{-1}$ at all; $\theta \eta$ increases $20-400$-fold as $c$ increases from 0 to $7 \mathrm{~g} / \mathrm{L}$; $(2)$ for the largest probes $(d$ $=189,282,455 \mathrm{~nm}), \theta \eta$ is a constant to within a factor of 2 of its average value. On the other hand, consider the fast mode [Fig. 3(b)]: for spheres of all sizes $\theta_{f} \eta$ increases 100 - to 400 -fold as $c$ is increased from 0 to $7 \mathrm{~g} / \mathrm{L}$. Therefore, of the four mode/probe-size combinations only the slow mode of the large probes shows close compliance with Stokes-Einsteinian behavior $(\theta \eta \approx$ const). In other words, at long times the relaxation of probes that are larger than the chain dimensions follows the macroscopic viscosity $\eta$ of the polymer solution. For the fast mode of large probes, and the slow and the fast modes of small spheres, probe motions at elevated polymer concentrations are much faster than motions that one would expect on the basis of the macroscopic solution viscosity.

\section{Mode-Scattering Vector Dependences}

Second, we consider the dependences of the fitting parameters on the scattering vector. Figure 4(a) shows the $q$ dependence of $\theta$ for the slow mode of large probes. The relaxation rate $\theta$ clearly shows diffusive behavior, i.e., $\theta \sim a q^{2}$; there is no intercept at $q=0$. This diffusive behavior confirms our above suggestion on the origin of the large-probe slow mode, namely that the largeprobe slow mode describes a time scale so long that polymer : solvent internal modes have completely relaxed. To the probes, on this time scale the medium is a simple viscous fluid. Therefore, from the central limit theorem one can predict that probes perform simple Brownian motion, implying $\beta_{t}=1$ and $\theta \sim q^{2}$, as found experimentally.

Figure 4(b) shows the $q$ dependence of the intermediate time scale mode, which incorporates the large-probe fast mode and the small-probe slow mode. The slow mode of small probes shows ${ }^{20}$ a clear diffusive behavior with $\theta \sim a q^{2}$ and zero intercept as $q \rightarrow 0$. However, the fast mode of large probes has a very complicated $q$ behavior. As seen in Figure 4(b), $\theta_{f}$ of large probes is largely independent of $q$ at small angles $(q$ $<250 \mu \mathrm{m}^{-2}$ ), but has a linear dependence $\theta_{f}$ $\sim a q^{2}+b$ with nonzero intercept $b$ at large angles $\left(q>250 \mu \mathrm{m}^{-2}\right)$.

Finally, the $q$ dependence of the short time scale mode (the fast mode of small probes) is complex, ${ }^{20}$ having two linear regimes, namely $\theta_{f}$ 


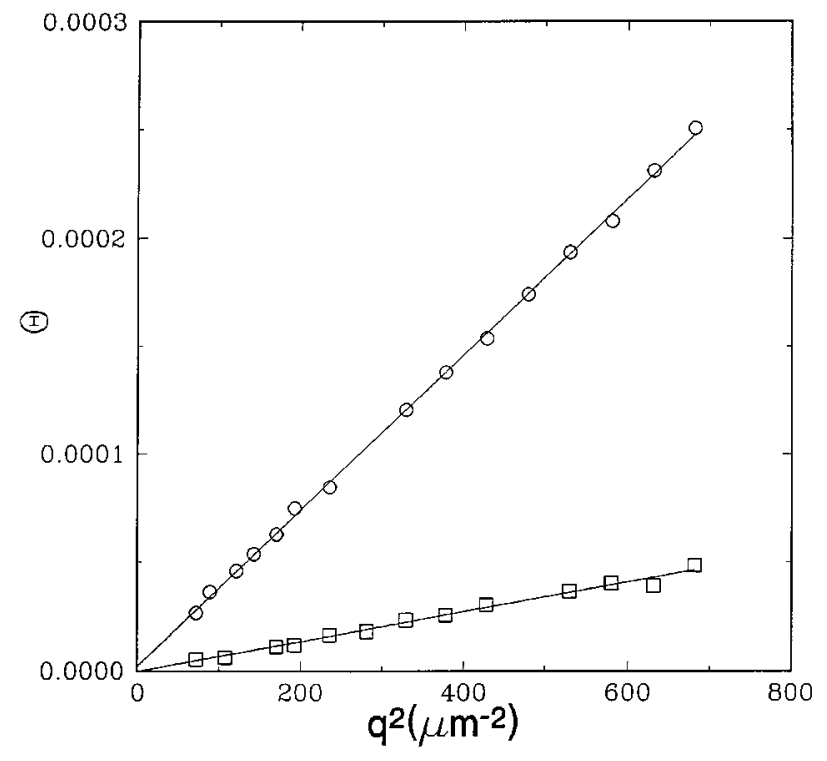

(a)

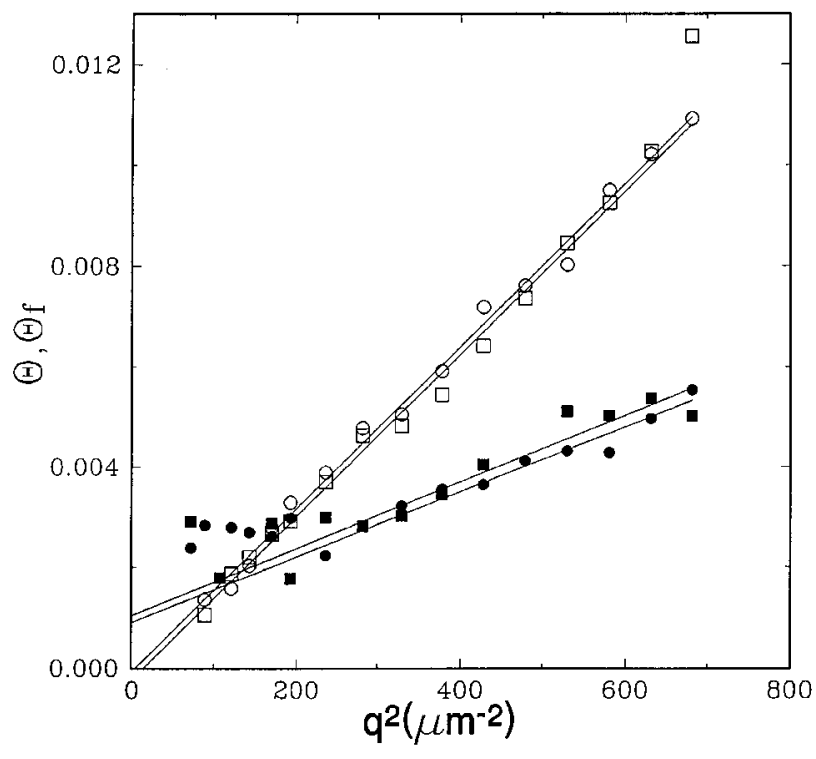

(b)

Figure 4. $q$-Dependence of the relaxation pseudorates for probes in HPC : water in different time scale regimes: (a) long time scale $\theta$ of large $189 \mathrm{~nm}$ probes in $2 \mathrm{~g} / \mathrm{L}(\bigcirc)$, and $5 \mathrm{~g} / \mathrm{L}(\square) \mathrm{HPC}$; (b) intermediate time scale $\theta$ of small $21 \mathrm{~nm}$ probes in $1 \mathrm{~g} / \mathrm{L}(\bigcirc), 4$ $\mathrm{g} / \mathrm{L}(\square) \mathrm{HPC}$, and $\theta_{f}$ of large $189 \mathrm{~nm}$ probes in $2 \mathrm{~g} / \mathrm{L}(\bullet), 5 \mathrm{~g} / \mathrm{L}(\boldsymbol{\square}) \mathrm{HPC}$. Solid lines are best linear fits. Point $(区)$ on (b) was omitted from the fit.

$\approx a q^{2}$ at large $q$ and $\theta_{f} \approx a_{1} q^{2}+b$ with $b$ $\neq 0$ at small $q$.

The fraction of the fast mode $A_{f}$ depends differently on $q$ for small and large probes. Figure 5 shows $q$ dependences of $A_{f}$ for large and small probes. For large probes, $A_{f}$ at small $q$ increases weakly with rising $q$, but is independent of $q$ at large $q$. The crossover in $A_{f}(q)$ between smalland large- $q$ behavior happens at the same $q$ as the crossover in $\theta(q)$ for large probes. The crossover is at $q^{2} \approx 250 \mu \mathrm{m}^{-2}$, equivalent to a crossover distance $q^{-1} \approx 63 \mathrm{~nm}, 63 \mathrm{~nm}$ also being the distance scale on which small- and largeprobe behaviors cross over. On the other hand, for small probes, $A_{f}$ decreases quasi-exponentially with increasing $q$; if one identifies the large-probe fast mode with the small-probe slow mode, the amplitude of this mode has similar $q$ dependence for large and small probes.

\section{Mode-Stretching Exponents}

Third, we consider the stretching exponents of three time scale regimes. The long time scale regime (large-probe slow mode) has ${ }^{20} \beta=1$. The intermediate time scale regime (large-probe fast and small-probe slow) have ${ }^{20} \beta$ and $\beta_{f}$ in the same range $(0.6,0.95) . \beta$ of small probes and $\beta_{f}$ of large probes both monotonically decrease ${ }^{20}$ with increasing $c$ from 0.95 near $c=0$ to 0.6 at $7 \mathrm{~g} / \mathrm{L}$ HPC. Also, $\beta$ of small probes and $\beta_{f}$ of large probes are both $q$ independent, ${ }^{20}$ even though $A_{f}(q)$ and $\theta_{f}(q)$ are very different for the smallprobe slow and large-probe fast modes. The short time scale regime (the small-probe fast mode) has $^{20}$ the smallest stretching exponent; $\beta_{f} \in(0.2$, $0.6)$. $\beta_{f}$ of small probes also decreases ${ }^{20}$ with increasing $c$, but has a nontrivial $q$ dependence (at low $q, \beta_{f}$ decreases with increasing $q$, at high $q, \beta_{f}$ is $q$ independent).

The aforementioned properties demonstrate that modes on the three time scales in general differ from each other. Physical interpretations for the intermediate and short time scale regimes will be advanced in the following sections. Here we will distinguish the properties of these two time scale modes and identify justifications, in addition to the similar time scale, for combining the large-probe fast mode and the small-probe slow mode into a single intermediate time scale regime.

First, consider the intermediate time scale regime. Two modes (the large-probe fast- and smallprobe slow-modes) compose the intermediate time 


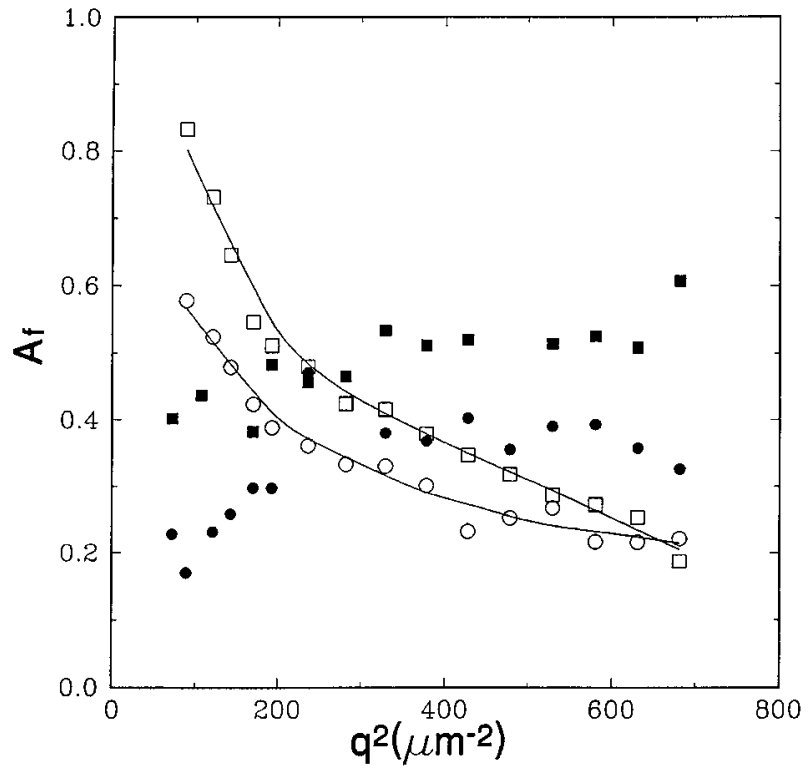

Figure 5. Amplitude fraction parameter $A_{f}$ of the fast mode from eq. (4) as a function of $q^{2}$ for 21-nm probes in $1 \mathrm{~g} / \mathrm{L}(\bigcirc)$ and $4 \mathrm{~g} / \mathrm{L}(\square) \mathrm{HPC}$, and for $189-\mathrm{nm}$ probes in $2 \mathrm{~g} / \mathrm{L}(\bigcirc)$ and $5 \mathrm{~g} / \mathrm{L}(\boldsymbol{\square})$ HPC. Lines are drawn to guide the eye.

scale. The large-probe fast and the small-probe slow modes differ significantly only in their $q$ dependences. This difference in $q$ dependence can be rationalized as the effect of probe size on the coupling of probe motions to the chain relaxations. In all other physical properties revealed by our previous study ${ }^{20}$ these two modes are similar. Particular similarities between the largeprobe fast mode and the small-probe slow mode include: (1) stretched-exponential (not simple-exponential) observed spectral lineshapes; (2) $c$ independences of $\theta$ for small probes and $\theta_{f}$ for large probes (within a factor of 2); (3) noncompliance of mode pseudorates with a Stokes-Einstein equation (large probes: $\theta_{f} \eta \neq$ const; small probes: $\theta \eta$ $\neq$ const); (4) very weak (large probes) or no (small probes) $d$ dependence of $\theta_{f}$ or $\theta$, respectively, and (5) $\beta_{f}$ (large probes) and $\beta$ (small probes) both fall with increasing $c$ from 0.95 in pure solvent to 0.6 at $7 \mathrm{~g} / \mathrm{L}$ of polymer.

Figure 6 compares $\tau$ of small probes and $\tau_{f}$ of large probes. Solid lines are drawn through the data on small probes $(\tau)$ to guide the eye and to help distinguish $\tau$ of small probes from $\tau_{f}$ of large probes. If the $c$ dependences of $\theta$ (and $\beta$ ) and $\theta_{f}$ (and $\beta_{f}$ ) are the same for these two modes, then the $c$ dependences of the true relaxation rates $\tau$ and $\tau_{f}$ should be the same. $\tau$ and $\tau_{f}$ indeed both increase with rising $c$. The decay pseudorates $\theta$ and $\theta_{f}$ are concentration independent; $\tau$ and $\tau_{f}$ inherit their dependences on $c$ exclusively from $\beta$ and $\beta_{f}$.

Second, consider the short time scale regime, i.e., the small-probe fast mode. Several properties of this mode distinguish it from others. In addition to having the largest relaxation pseudorate $\theta_{f}$, this mode has the smallest stretching exponent $\beta_{f}$. This combination of large $\theta_{f}$ and small $\beta_{f}$ makes this mode very broad, so that $\tau_{f}>\tau$ with a very sharp initial decay. Other properties of this mode appear to be similar to properties of the intermediate time scale mode. In particular, the short time scale mode shows non-Stokes-Einsteinian behavior; to first approximation, $\theta_{f}$ is $c$ independent. The stretching exponent $\beta_{f}$ of the short time scale regime decreases with increasing $c$, just as the stretching exponents of the intermediate time scale mode do. However, the relaxation time $\tau_{f}$ of the short time scale regime appears to be $c$ independent, in contrast to the $c$ dependence of $\tau$ and $\tau_{f}$ of the intermediate time scale mode.

Summarizing, we have analyzed the properties of the four mode/probe-size combinations and have identified three physical time scales of probe motion. The large-probe slow mode corresponds to the longest time scale. The properties of this mode

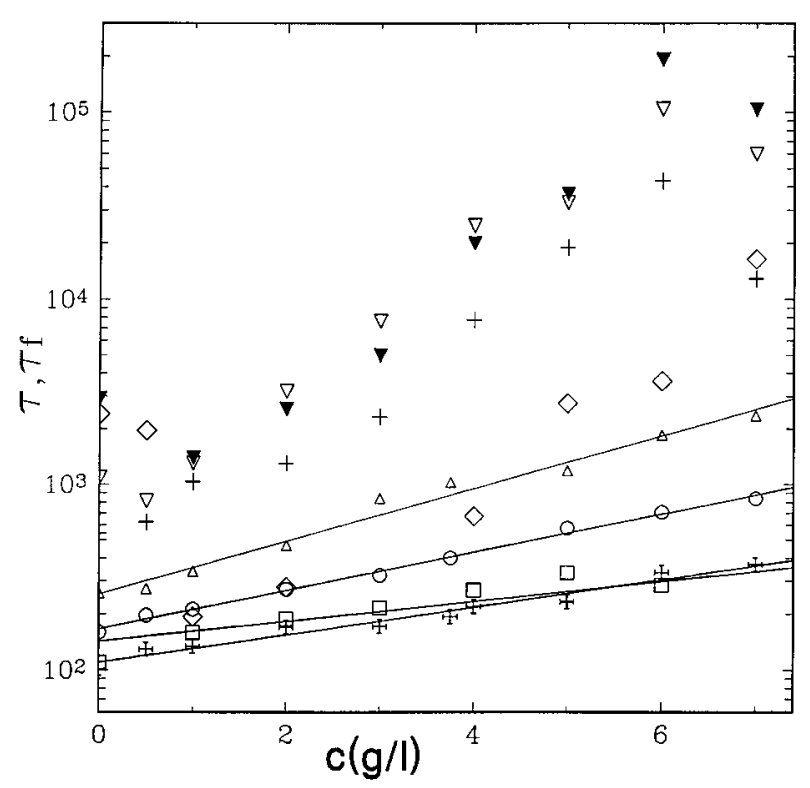

Figure 6. Concentration dependences of relaxation times: $\tau=\theta^{-1 / \beta}$ for the slow mode of small probes with diameter $87(\diamond), 189(+), 282(\nabla)$, and $455(\nabla) \mathrm{nm}$. Units of $\tau$ and $\tau_{f}$ are $(\mu S)$. Solid lines are drawn to guide the eye through the data on small probes $(\tau)$. 
indicate that on this time scale the polymer solution behaves as a simple viscous fluid, and spheres sample the macroscopic viscosity of the solution. The intermediate time scale incorporates the small-probe slow mode and large-probe fast mode. These two modes have similar physical properties, except for their $q$ dependences, differences which can be attributed to a probe-size effect. The small-probe fast mode corresponds to the shortest time scale. Most properties of this mode are very different from other modes, except for a few properties that are similar to the intermediate time scale regime, notably the nonStokes-Einsteinian behavior and, to first approximation, some but not all $q$ dependences.

\section{COUPLING/SCALING ANALYSIS}

The coupling model of $\mathrm{Ngai}^{28-32}$ is a general model for "the dynamics of constrained, interacting systems." ${ }^{21}$ The coupling model has been successfully applied to a variety of complex physical systems. ${ }^{32}$ Even though the coupling model for real complex systems has no theoretical derivation from the first principles yet, from simulating simple systems with idealized Hamiltonians there are several encouraging theoretical results $^{33-35}$ that directly support basic elements of the coupling model. The most recent examples of such theoretical modeling via computer dynamics simulations are (1) the relaxation of interacting arrays of coupled nonlinear oscillators, ${ }^{34}$ and (2) the Fermi acceleration problem with added nonlinearity. ${ }^{35}$ In both theoretical examples, the basic principles of classical mechanics were used to estimate the effects of nonlinearity on the relaxational processes. Both simulations yielded the coupling model predictions for nonlinearly coupled many-body systems.

The coupling model looks at a system as a combination of "basic units" interacting nonlinearly with each other. The simple coupling model considers two time scales, separated by a crossover time $t_{c}$. At $t<t_{c}$, "basic units" are assumed to relax independently from each other; their relaxation can be described by a correlation function

$$
\phi(t)=\exp \left(-t / \tau_{0}\right), \quad t<t_{c} .
$$

$\tau_{0}$ is the characteristic time for unconstrained relaxation. At $t>t_{c}$, cooperative constrains be- tween "basic units" become important, and the degree $n(0 \leq n<1)$ of coupling between basic units should be accounted for in $\phi(t)$, so that

$$
\phi(t)=\exp \left(-(t / \tau)^{(1-n)}\right), \quad t>t_{c} .
$$

Here, $\tau$ is the characteristic time for relaxations under constrains. There is also a continuity condition on $\phi(t)$ at $t=t_{c}$, which yields the requirement $\tau=\left[t_{c}^{-n} \tau_{0}\right]^{1 / 1-n}$. The coupling model is not specific on which interactions (e.g., hydrodynamic, topological) create the cooperative constraints.

The coupling model successfully predicts some experimental results on probe diffusion in polymer solutions ${ }^{11,15,17}$ and on polymer self-diffusion. ${ }^{36-38}$ First, stretched-exponential functions of $\phi(t)$ describe well $g^{(1)}(t)$ of many polymer systems. ${ }^{11,15,17,39-41}$ In terms of our notation, $\beta=1$ $-n$. Second, it was shown by Ngai and Phillies ${ }^{21}$ that the phenomenological concentration dependence of $\beta^{11,15,17,40,42}$ is often consistent with coupling model predictions for $1-n$. Third, the coupling model predicts ${ }^{21}$ that the relaxation time $\tau$ depends on $q$ as $\tau \equiv \theta^{-1 / \beta_{q}} \sim q^{-2 /(1-n)}$. This $q$ dependence was seen experimentally ${ }^{15}$ in our laboratory.

Recently, Ngai and Phillies ${ }^{21}$ advanced coupling/scaling arguments providing one with an additional two ways to extract the degree of coupling $n$ from the phenomenology. The first way is the use of the concentration dependence of the probe diffusion coefficient $D_{s}$, where coupling/ scaling gives the degree of coupling $n_{D}$, namely

$$
\begin{aligned}
& \beta_{c} \equiv 1-\eta_{D} \\
& =\left[\frac{3 \nu}{2}+\frac{(3 \nu-1)\left(\ln \left(D_{s}\left(c^{*}\right)\right)-\ln \left(D_{s}(c)\right)\right)}{2 \ln \left(c / c^{*}\right)}\right]^{-1} .
\end{aligned}
$$

Here, $c^{*}$ is a nominal overlap concentration, and $\nu$ from $R_{g} \sim M^{\nu}$ relates the radius of gyration $R_{g}$ of a polymer to its molecular weight $M$. Equation (8) is most plausible for nondilute solutions, i.e., for $c \geq 2 c^{*}$.

The second way is the use of the concentration dependence of $\eta$, where coupling/scaling gives the degree of coupling $n_{\eta}$, namely

$$
\begin{aligned}
& \beta_{\eta} \equiv 1-n_{\eta} \\
& =\left[\frac{3 \nu}{2}+\frac{(3 \nu-1)\left(\ln (\eta(c))-\ln \left(\eta\left(c^{*}\right)\right)\right)}{2 \ln \left(c / c^{*}\right)}\right]^{-1} .
\end{aligned}
$$


Equations (8) and (9) provide two paths for obtaining the degree of coupling $n \equiv 1-\beta$. Two additional independent ways for obtaining the degree of coupling are: (1) $\beta_{t}$ from the $t$ dependence of $g^{(1)}(t)$, and (2) $\beta_{q}$ from the $q$ dependence of $g^{(1)}(t)$. An obvious way to test the cou$\mathrm{pling} / \mathrm{scaling}$ model is to compare $\beta_{t}, \beta_{q}, \beta_{c}$, and $\beta_{\eta}$ for experimental data on one system. If all four $\beta$ agree with each other, then the model is selfconsistent. Phillies and $\mathrm{Ngai}^{21}$ were able to demonstrate the success of the model for probe diffu$\operatorname{sion}^{17}$ in $300 \mathrm{kDa}$ HPC solutions. They demonstrated that $\beta_{c}, \beta_{q}$, and $\beta_{t}$ from $g^{(1)}(t)$, and $\beta_{\eta}$ from $\eta(c)$ agree with each other.

Recently, we ${ }^{22}$ applied the coupling/scaling model to the data of ref. 20 on $M=1 \times 10^{6} \mathrm{Da}$ HPC. A simple repeat of the coupling/scaling analysis was impossible, because the spectra of ref. 20 have two relaxational modes, not one. Ref. 20 's spectra do not imply a single diffusion coefficient $D_{p}$ that could be substituted in eq. (8). The two modes appear to be very different in their physical nature. In particular, large probe diffusion requires whole-chain center-of-mass motions, but small probe diffusion can be accommodated by local chain motions. As shown in the previous section, the slow mode of large probes reflects a simple Brownian motion of the probes in the polymer matrix; regarding other modes, ref. 22 proposed that the intermediate time scale mode involves probe motions coupled to chain local motions.

Because the spectral lineshape is bimodal, we cannot apply the simple coupling/scaling model ${ }^{21}$ to the whole relaxation. However, very recently Ngai and Rendell ${ }^{43}$ have shown an approach to applying the coupling model to a much more complicated relaxation, namely diffusion in a concentrated solution of hard colloidal spheres as studied by Segre and Pusey. ${ }^{44}$ In these systems, the light-scattering spectrum is composed of a shorttime pure exponential regime, an intermediatetime stretched exponential-in-time regime, and a long-time pure exponential regime. Ngai and Rendell ${ }^{43}$ concluded that the coupling model procedures are applicable to this systems, in that "there is good agreement between [the model] and experiment."

In contrast to the system described by Ngai and Rendell, ${ }^{43}$ in which the functional form of the relaxation depends on time, so that three time regimes exist, here we have bimodal spectra that are represented as a sum of two independent modes. The coupling ansatz naturally leads to such spectra if the system contains several groups of fundamental units that are strongly coupled within each group, but that are very weakly coupled between groups. We ${ }^{22}$ therefore, analyzed each mode separately using coupling/scaling, so as to determine which modes are described by the coupling model.

Two sets of coupling coefficients, namely $\beta_{t}$ and $\beta_{c}$ for the slow mode, and $\beta_{f t}$ and $\beta_{f c}$ for the fast mode, were obtained ${ }^{22}$ from the measured $g^{(1)}(t)$ and eq. (8). $\beta_{\text {eta }}$ is calculated using eq. (9) from $\eta(c)$, so $\beta_{\eta}$ is the same for all modes. Dealing separately with each mode, we can compare $\beta_{t}$, $\beta_{c}, \beta_{f t}$, and $\beta_{f c}$ to $\beta_{\eta}$ to infer the coupling behavior of each mode. $\beta_{\eta}$ is very important in these comparisons because it reflects the motion of complete chains (a matrix property unrelated to probe radius), and because it is calculated directly from coupling/scaling analysis ${ }^{21}$ without auxiliary assumptions.

We found ${ }^{22}$ that some modes follow the coupling/scaling model, while others do not. The slow mode of large probes and the fast mode of small probes do not follow coupling/scaling predictions, so these modes are not considered further here. The intermediate time scale regime (small-probe slow mode and large-probe fast mode) obeys the coupling/scaling model. We consider here this time scale mode in detail.

Figures 7(a) (small-probe slow mode) and 7(b) (large-probe fast mode) show exemplary results of the coupling/scaling analysis for the small-probe slow mode and the large-probe fast mode, respectively, comparing $\beta_{t}$ and $\beta_{c}$ (or $\beta_{f c}$ ) with $\beta_{\eta}$ from the solution viscosity. To apply eqs. (8)-(9), one needs a nominal overlap concentration $c^{*}$ and scaling exponent $\nu$. From ref. 27, for 1 MDa HPC $c^{*}=1 /[\eta]$ is $1.4 \mathrm{~g} / \mathrm{L}$. We tried three plausible values for $\nu$, namely $0.5,0.55$, and 0.6 . The $\beta_{\eta}$ and $\beta_{c}$ calculated with a specific $\nu$ are denoted $\beta_{\eta, \nu}$ and $\beta_{c, \nu}$, respectively. Near $c^{*}, \beta_{\eta, \nu}$ is scattered because of a numerical artifact; at $c^{*}$, eq. (9) approaches $\ln (1) / \ln (1)$. For $c>c^{*}, \beta_{\eta, \nu}$ decreases smoothly with increasing $c$.

From Figure 7(a), the small-probe slow mode has $\beta_{t} \approx \beta_{c, 0.55}$ at $c>3-4 \mathrm{~g} / \mathrm{L}$, which is in agreement with the coupling/scaling model. Furthermore, at $c>3-4 \mathrm{~g} / \mathrm{L}, \beta_{t} \approx \beta_{\eta, 0.5}$. The concentration threshold of 3-4 g/L, above which the model applies, is more than twice $c^{*}$. The fact that $\beta_{t} \approx \beta_{c, 0.55}$ is true only at $c>2 c^{*}$, is consistent with expectations of ref. 21 that coupling/scaling is more likely to be valid in a nondilute solution. The finding $\beta_{t} \approx \beta_{c, 0.55} \geq \beta_{\eta, 0.55}$ 


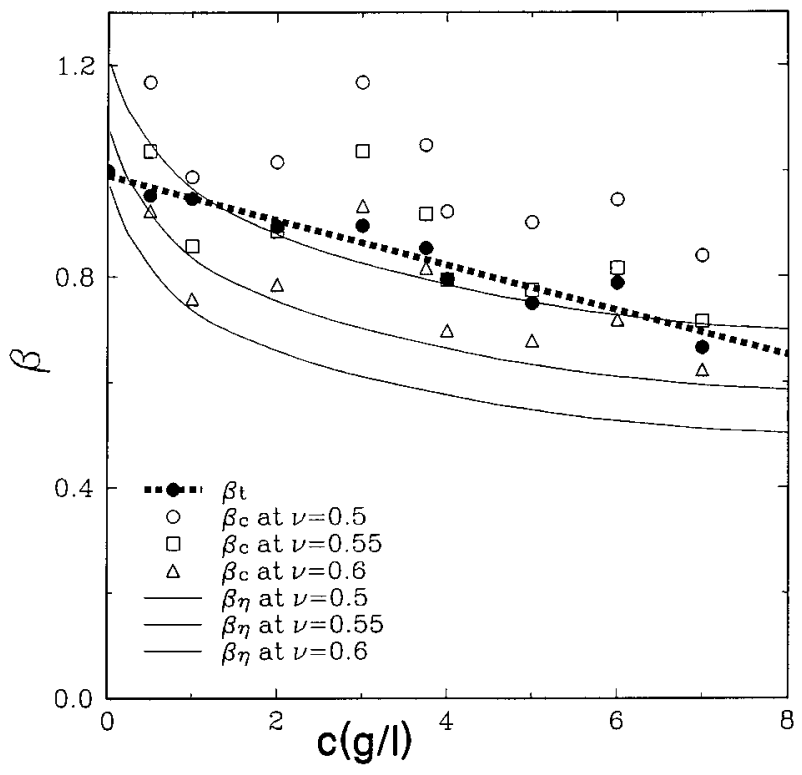

(a)

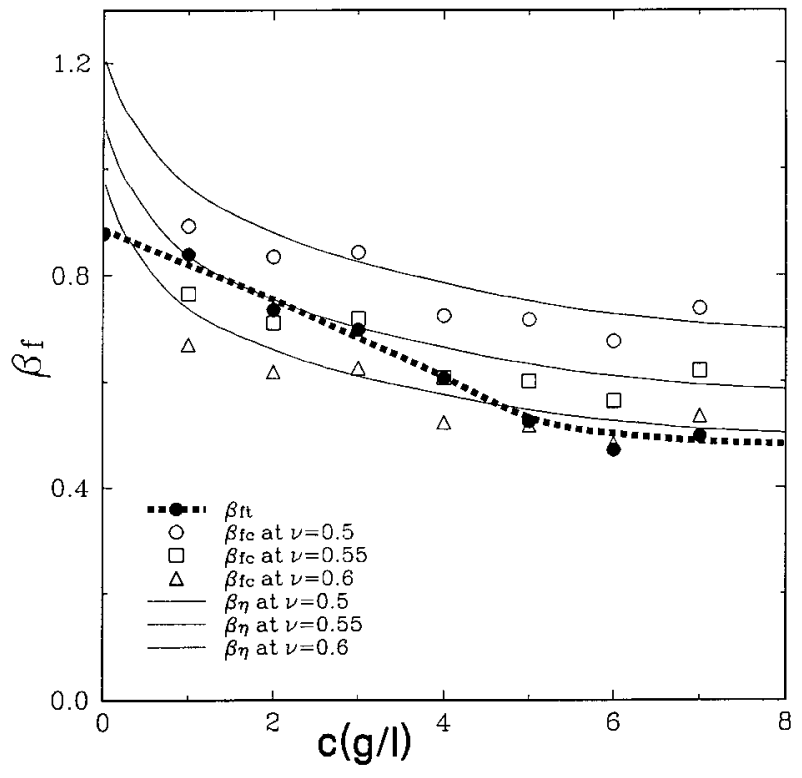

(b)

Figure 7. Concentration dependences of the stretching exponents for the intermediate time scale regime: (a) the small-probe $(14 \mathrm{~nm})$ slow-mode exponents $\beta_{t}$ from $g^{(1)}(t)$ via eq. (4), and $\beta_{c}$ from $\theta$ via eq. (8) (for three values of $\nu$ ); and (b) the large-probe (455 $\mathrm{nm})$ fast-mode exponents $\beta_{f t}$ from $g^{(1)}(t)$, and $\beta_{f c}$ from $\theta_{f}$ (for three values of $\nu$ ). Parts (a) and (b) also show $\beta_{\eta}$ from $\eta^{27}$ via eq. (9) (solid lines corresponding, top to bottom, to 0.5, 0.55, and 0.6. Dashed lines, drawn to guide the eye, correspond to $\beta_{c}(c)$ and $\beta_{f c}(c)$.

is not exactly the result one would expect from a self-consistent model. ${ }^{21}$ However, the inequality between $\beta_{\eta}$ and $\beta_{t}$ at $c>2 c^{*}$ for small probes is consistent with coupling/scaling predictions. $\beta_{\eta}$ describes relaxations of whole chains; $\beta_{t}$ and $\beta_{c}$ describe probe motions. Under the coupling mod$\mathrm{el},{ }^{21}$ the strength of dynamic constraints is reflected in the degree of coupling $n$. Because viscous flow moves whole chains, while small probes with $d \leq R_{g}$ can diffuse while displacing only part of the neighboring chain, polymer chain motions encounter larger dynamic constraints than probe motions, implying $\beta_{t} \approx \beta_{c}>\beta_{\eta}$, as found experimentally. Therefore, Figure 7(a) largely supports the coupling/scaling model predictions. Figure 7(b) shows that for the large-probe fast mode $\beta_{t} \approx \beta_{c, 0.6} \approx \beta_{\eta, 0.6}$, in the excellent agreement with the coupling/scaling model.

\section{DISCUSSION}

Ref. 20 found four relaxation modes in optical probe diffusion spectra. We have argued here that these four modes can be rationally divided into three physical regimes: (1) a long time scale re- gime; (2) an intermediate time scale regime; and (3) a short time scale regime. The rationale for dividing these modes into three regimes is based on their physical properties, notably the time scale of the mode relaxation (the four modes decay on only three different time scales), and the value of the stretching parameters $\beta$ and $\beta_{f}$ [only three regimes: $\beta=1, \beta, \beta_{f} \in(0.6,1)$, and $\beta, \beta_{f}$ $\epsilon(0.2,0.6)]$. Table I summarizes these differences.

What is the physics underlying the time scale regimes? We know the physical nature of the longest time scale regime. As noted above, the large-probe slow mode describes the motion of probes on time scale so long that the solution acts as a sample viscous liquid. Correspondingly, large probes perform Brownian motion with $\theta \sim q^{2}, \beta$ $=1$ and follow the Stokes-Einstein equation $(\theta \eta$ $\approx$ const).

Now we consider the properties of other two regimes. We start with the two modes of the intermediate time scale regime. As mentioned in previous sections, the small-probe slow mode and the large-probe fast mode have common properties, except for their $q$ dependences. This difference in $q$ dependences can be rationalized as the 
Table I. Properties of the Four Relaxational Modes for Large and Small Probe Diffusion in $1 \mathrm{MDa}$ HPC

\begin{tabular}{|c|c|c|}
\hline \multirow[b]{2}{*}{ Probe Size } & \multicolumn{2}{|c|}{ Mode } \\
\hline & Slow & Fast \\
\hline & $\theta \sim 10^{-3}-5 \times 10^{-6}$ & $\theta_{f} \sim 10^{-2}-3 \times 10^{-3}$ \\
\hline \multirow[t]{2}{*}{ Large } & $\beta=1$ & $\beta_{f} \in(0.6,0.95)$ \\
\hline & $\theta \sim \exp \left(-\alpha c^{\nu}\right)$ & $\theta_{f} \sim c^{0}($ within 2$)$ \\
\hline \multirow[t]{2}{*}{ probes } & $\theta \sim a q^{2}$ & $\theta_{f}\left(q^{2}\right)$-complicated \\
\hline & $\theta \downarrow$ with $d \uparrow, \alpha \sim d$ & $\theta_{f} \sim d^{0}$ (within 2$)$ \\
\hline \multirow[t]{4}{*}{$\left(d>R_{g}\right)$} & 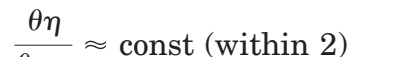 & $\underline{\theta_{f} \eta} \neq$ const \\
\hline & $\theta_{0} \eta_{0}$ & $\theta_{f 0} \eta_{0}$ \\
\hline & & $\begin{array}{l}\beta_{f} \downarrow \text { with } c \uparrow \\
\beta_{f} \sim q^{0}\end{array}$ \\
\hline & $\begin{array}{l}A_{f} \text { largely independent of } q \\
\frac{d \tau}{d c}>0\end{array}$ & $\begin{array}{l}A_{f} \text { largely independent of } q \\
\frac{d \tau_{f}}{d c}>0\end{array}$ \\
\hline \multirow[t]{2}{*}{$\begin{array}{l}\text { Coupling/scaling } \\
\text { (c/s) analysis }\end{array}$} & $\begin{array}{l}1 \equiv \beta_{t}>\beta_{c, \nu} \approx \beta_{\eta, \nu} \\
\mathrm{c} / \mathrm{s} \text { fails }\end{array}$ & $\begin{array}{l}\beta_{f t} \approx \beta_{f c, \nu} \approx \beta_{\eta, \nu} \\
\mathrm{c} / \mathrm{s} \text { successful }\end{array}$ \\
\hline & $\theta \sim 10^{-2}-3 \times 10^{-3}$ & $\theta_{f} \sim 5 \times 10^{-2}$ \\
\hline \multirow[t]{2}{*}{ Small } & $\beta \in(0.6,0.95)$ & $\beta_{f} \in(0.2,0.6)$ \\
\hline & $\theta \sim c^{0}$ & $\theta_{f} \sim c^{0}($ within 2$)$ \\
\hline probes & $\begin{array}{l}\theta_{f} \sim q^{2} \\
\theta_{f} \sim d^{0}(\text { within } 2)\end{array}$ & $\begin{array}{l}\theta_{f}(q) \text {-complicated } \\
\theta_{f} \sim d^{0}\end{array}$ \\
\hline \multirow{6}{*}{$\left(d<R_{g}\right)$} & $\theta \eta$ & $\theta_{f} \eta$ \\
\hline & $\overline{\theta_{0} \eta_{0}} \neq$ const & $\overline{\theta_{f 0} \eta_{0}} \neq=$ const \\
\hline & $\beta \downarrow$ with $c \uparrow$ & $\beta_{f} \downarrow$ with $c \uparrow$ \\
\hline & $\beta \sim q^{0}$ & $\beta \sim q^{0}$ \\
\hline & $A_{f}(q)$-very strong & $A_{f}(q)$-very strong \\
\hline & $\frac{d \tau}{d c}>0$ & $\tau_{f} \sim c^{0}$ \\
\hline $\begin{array}{l}\text { Coupling/scaling } \\
\text { (c/s) analysis }\end{array}$ & $\begin{array}{l}\beta_{t} \approx \beta_{c, 0.55} \geq \beta_{\eta, 0.55} \\
\text { c/s largely successful }\end{array}$ & $\begin{array}{l}\beta_{f t} \leq \beta_{\eta, \nu} \leq \beta_{f c, \nu} \\
\text { c/s fails }\end{array}$ \\
\hline
\end{tabular}

All notation defined in text.

effect of probe size on the coupling of probe motions to polymer relaxations. On this time scale, large probes are fully coupled to polymer solution motions at all but very long distances (low $q$ ), because at long distances probes have a chance to decouple from chain internal motions and experience the long wavelength shear viscosity. Correspondingly, $A_{f}$ is independent of $q$ at all but very low $q$. However, the $q$ dependence is very marginal: $A_{f}$ is independent of $q$ over $85-90 \%$ of the studied $q$ scale. Small probes are small enough $(d$ $<2 R_{g}$ ) to be sensitive to the solution structure, in addition to sensing the shear viscosity of the medium. As a result, the spatial dimensions of the chain become important to the probe and $A_{f}$ (as well as $\theta_{f}$ ) show a strong dependence on $q$.
The above-mentioned $q$ dependence differences between the two modes of the intermediate time scale regime can, therefore, be rationalized in terms of the probe coupling to the polymer. The two modes of the intermediate time scale regime also have the common properties, mentioned in the Results section. Can these properties also be rationalized in terms of the probe motions coupled to polymer relaxations?

Experimental phenomenology supportive of an interpretation of intermediate mode properties as arising from motions of probes coupled to polymer motions includes: (a) the observed lineshape is a stretched exponential, not a simple exponential in $t$; (b) $\beta$ (small spheres) and $\beta_{f}$ (large spheres) fall with increasing $c$, consistent with the coupling 
model expectation that the coupling coefficient $n$ $=1-\beta$ increases with increasing $c$, because the coupling of probes to polymers is stronger at higher polymer concentrations; and (c) both modes of the intermediate time scale largely comply with the coupling/scaling prediction $\beta_{t}$ $=\beta_{c}=\beta_{\eta}$, namely for large spheres $\beta_{f t} \approx \beta_{f c, \nu}$ $\approx \beta_{\eta, \nu}$, while for small spheres $\beta_{t} \approx \beta_{c, \nu}$, (though $\beta_{t}, \beta_{c, \nu} \geq \beta_{\eta, \nu}$ ). The above three properties were directly predicted by the coupling/ scaling model. ${ }^{21}$ Other phenomenological findings that are consistent with the idea that the intermediate time scale mode reflects the motion of probes, coupled to polymer relaxations, include: (1) $\theta$ (small spheres) and $\theta_{f}$ (large spheres) are practically independent of $d$ (within a factor of 2). A mode whose dynamics is largely determined by chain motion, in which probes are passive witnesses to the chain-chain relaxations, could have a relaxation rate independent of $d$; (2) the $q$ dependence depends on the probe size, as explained above; (3) neither $\theta$ nor $\theta_{f}$ tracks $\eta^{-1}$. The relaxation pseudorates of the two modes do not simply reflect the macroscopic viscosity of the solution, because probes, in addition of being sensitive to long wavelength shear viscosity, also are coupled to polymer relaxations; (4) $\tau$ (small probes) and $\tau_{f}$ (large probes) depend on $c$. A mode with a strong coupling to polymer relaxations should have a concentration-dependent relaxation rate, because at higher $c$ dynamic constraints are stronger, so the relaxations are slower; and (5) the concentration dependence of the large-probe $\tau_{f}$ is stronger than the concentration dependence of the smallprobe $\tau$, consistent with the expectation that larger probes couple more strongly to polymer motions than do smaller probes.

We finish our mode analysis by considering the small-probe fast mode. We propose that at very short times probes sample local chain relaxations. The short time scale is sampled by the fast mode of small probes. These probes are much smaller than chains, so at our concentration a single small probe is unlikely to be in contact with more than one or two chains at a time. At short distances and times, chain interactions have not yet fully established themselves, so $\theta_{f}$ should be relatively independent of $c$, as observed experimentally. If probe motions are dominated by the motions of one neighboring chain, $\theta_{f}$ will be determined by internal chain dynamics of a single chain, and will be relatively independent of $d$, also as observed experimentally.
In summary, the four observed relaxation modes in optical probe diffusion spectra were shown to comprise three physical time scale regimes: (1) a long time scale regime, consisting of the large-probe slow mode; (2) an intermediate time scale regime incorporating the small-probe slow mode and the large-probe fast mode; and (3) a short time scale regime represented by the small-probe fast mode.

The proposed physical picture underlying these time scale regimes is based on the assumption that probe relaxations reflect motions of polymer chains that occur on the three physical time scales. On the longest time scale, all polymer modes have decayed and the solution behaves like a viscous fluid. In this time scale, probes sample the viscous fluid. The coupling/scaling model does not work for this regime. On the intermediate time scale, we propose that probe motions are coupled to polymer relaxations. The coupling/scaling model largely succeeds in describing this regime. The shortest time scale is not described by the coupling/scaling model. We propose that on this time and distance scale local motions of single chains are significant, and that probes sample local chain relaxations of individual chains.

Partial support of this work by the National Science Foundation under Grant DMR94-23702 is gratefully acknowledged.

\section{REFERENCES}

1. T. P. Lodge, N. Rotstein, and S. Prager, Adv. Chem. Phys., 79, 1 (1991).

2. D. N. Turner and F. R. Hallett, Biochem. Biophys. A, 451, 305 (1976).

3. T.-H. Lin and G. D. J. Phillies, J. Phys. Chem., 86, 4073 (1982).

4. T.-H. Lin and G. D. J. Phillies, J. Colloid Interface Sci., 100, 82 (1984).

5. T.-H. Lin and G. D. J. Phillies, Macromolecules, 17, 1686 (1984).

6. G. S. Ullmann and G. D. J. Phillies, Macromolecules, 16, 1947 (1983).

7. L. M. Wheeler and T. P. Lodge, Macromolecules, 22, 3399 (1989).

8. J. Won, C. Onyenemezu, W. G. Miller, and T. P. Lodge, Macromolecules, 27, 7389 (1994).

9. L. M. Wheeler and T. P. Lodge, Macromolecules, 19, 2983 (1986).

10. R. Furukawa, J. L. Arauz-Lara, and B. R. Ware, Macromolecules, 24, 599 (1991).

11. G. D. J. Phillies, G. S. Ullmann, K. Ullmann, and T.-H. Lin, J. Chem. Phys., 82, 5242 (1985). 
12. M. B. Mustafa and P. S. Russo, J. Colloid Interface Sci., 129, 240 (1989).

13. Z. Bu and P. S. Russo, Macromolecules, 27, 1187 (1994).

14. W. Brown and R. Rymden, Macromolecules, 19, 2942 (1986).

15. G. D. J. Phillies, C. Richardson, C. A. Quinlan, and S.-Z. Ren, Macromolecules, 26, 6849 (1993).

16. G. D. J. Phillies and D. Clomenil, Macromolecules, 26, 167 (1993).

17. G. D. J. Phillies and M. Lacroix, J. Phys. Chem. B, 101, 39 (1997).

18. G. D. J. Phillies, J. Chem. Phys., 60, 983 (1974).

19. G. D. J. Phillies, Biopolymers, 14, 499 (1975).

20. K. A. Streletzky and G. D. J. Phillies, J. Chem. Phys., 108, 2975 (1998).

21. K. L. Ngai and G. D. J. Phillies, J. Chem. Phys., 105, 8385 (1996).

22. K. A. Streletzky and G. D. J. Phillies, Coupling Analysis of Probe Diffusion in High Molecular Weight Hydroxypropylcellulose, submitted.

23. G. D. J. Phillies, J. Phys. Chem., 96, 10061 (1992).

24. J. H. Noggle, Physical Chemistry on a Microcomputer, Little, Brown and Company, Toronto, 1985.

25. M. Doi and S. F. Edwards, The Theory of Polymer Dynamics, Oxford University Press, Oxford, 1986.

26. P.-G. de Gennes, Scaling Concepts in Polymer Physics, Cornell University Press, Ithaca, NY, 1979.

27. G. D. J. Phillies and C. A. Quinlan, Macromolecules, 28, 160 (1995).

28. K. L. Ngai, A. K. Rajagopal, and S. Teiler, J. Chem. Phys., 88, 5086 (1988).
29. K. L. Ngai and R. W. Rendell, J. Molecular Liquids, 56, 199 (1993).

30. K. L. Ngai, R. W. Rendell, A. K. Rajagopal, and S. Teiler, Ann. NY Acad. Sci., 484, 150 (1985).

31. A. K. Rizos, T. Jian, and K. L. Ngai, Macromolecules, 28, 517 (1995).

32. K. L. Ngai, in Disorder Effects in Relaxational Processes, edited by R. Richert and A. Blumen, Springer, Berlin, 1994, pp. 89-150.

33. K. Y. Tsang and K. L. Ngai, Macromol. Chem. Macromol. Symp., 90, 95 (1995).

34. K. Y. Tsang and K. L. Ngai, Phys. Rev. E, 54, R3067 (1996).

35. K. Y. Tsang and K. L. Ngai, Phys. Rev. E, 56, R17 (1997).

36. J. Colmenero, A. Arbe, and A. Alegria, Phys. Rev. Lett., 71, 2603 (1993).

37. H. Walderhaug, B. Nystrom, F. K. Hansen, and B. Lindman, J. Phys. Chem., 99, 4672 (1995).

38. B. Nystrom, H. Walderhaug, and F. K. Hansen, $J$. Phys. Chem., 97, 7743 (1993).

39. B. Nystrom, J. Roots, A. Carlsson, and B. Lindman, Polymer, 33, 2875 (1992).

40. B. Nystrom, K. Thuresson, and B. Lindman, Langmuir, 11, 1994 (1995).

41. W. Brown and T. Nicolai, Macromolecules, 27, 2470 (1994).

42. B. Nystrom and B. Lindman, Macromolecules, 28, 967 (1995).

43. K. L. Ngai and R. W. Rendell, Philos. Mag. B, 77, 621 (1998).

44. P. N. Segre and P. N. Pusey, Phys. Rev. Lett., 77, 771 (1996). 